Pacific Journal of Mathematic 


\section{THE SPHERICAL CURVATURE OF A HYPERSURFACE IN EUCLIDEAN SPACE}

T. K. Pan

1. Introduction. Let $V_{n}$ be a hypersurface immersed in a Euclidean space $S_{n+1}$. Let $P$ be a point of $V_{n}$ corresponding to the point $P^{\circ}$ of the hyperspherical representation $G_{n}$ of $V_{n}$. Let $V$ denote the extension of a region $\phi$ of $V_{n}$, and $V^{\prime}$ the extension of the corresponding hyperspherical region $\phi^{\prime}$ of $G_{n}$. If the region around $P$ tends to zero, the ratio $V^{\prime} / V$ tends to a limit $\Gamma$, which is called the spherical curvature of $V_{n}$ at $P[1, \mathrm{pp} .258-261]$. It is found that $\Gamma=|\Omega / g|$, where $g=\left|g_{i j}\right|$ and $\Omega=\left|\Omega_{i j}\right|$ are respectively the determinants of the coefficients of the first and the second fundamental forms of $V_{n}$. In this note, some properties of the spherical curvature are studied, and new interpretations of the Gaussian curvature are derived.

The notation of Eisenhart [2] will be used for the most part.

2. Some properties. Let a real and analytic hypersurface $V_{n}$ be defined by

$$
y^{\alpha}=y^{\alpha}\left(x^{1}, \ldots, x^{n}\right) \quad(\alpha=1, \cdots, n+1),
$$

referred to a Cartesian coordinate system $y^{\alpha}$ in a Euclidean space $S_{n+1}$. Let a vector-field $v$ in $V_{n}$ be defined by

$$
v^{\alpha}=p^{i} \partial y^{\alpha} / \partial x^{i} \quad(i=1, \cdots, n),
$$

where the $v^{a}$ are real and analytic functions of the $x^{i}$. Let $C$ be a curve of $V_{n}$. The normal curvature vector of $v$ with respect to $C$ at $P$ is defined as the normal component of the derived vector of the vector-field $v$ along $C$ at $P$ [3]. Let $\kappa$ denote a nonzero extreme value of the magnitudes of the normal curvature vectors of $v$ with respect to all curves of $V_{n}$ at $P$. Then $\kappa$, which is called a principal curvature of $v$ at $P$, is defined by

$$
\left|\Psi_{i j}-\kappa^{2} g_{i j}\right|=0
$$

Received May 13, 1952.

Pacific J. Math. 3 (1953), $461-466$ 
where

$$
\Psi_{i j}=\Omega_{i k} \Omega_{j l} p^{k} p^{l} / g_{k l} p^{k} p^{l}
$$

Since $\left\|\Psi_{i j}\right\|$ is of rank 1 , there is one such extreme corresponding to a vectorfield $v$. Its value is evidently equal to

$$
\kappa=\left(\Psi_{i j} g^{i j}\right)^{1 / 2}=\left(H_{i j} p^{i} p^{j} / g_{i j} p^{i} p^{j}\right)^{1 / 2},
$$

where $H_{i j}$ is the fundamental tensor of the hyperspherical representation $G_{n}$.

The extreme of the principal curvature of a vector-field $v$ at $P$, as the field varies, is defined by

$$
\left|H_{i j}-\kappa^{2} g_{i j}\right|=0
$$

There are $n$ such extremes $\bar{\kappa}_{i}$ corresponding to the principal directions for the tensor $H_{i j}$. Their product is found to be

$$
\prod_{i=1}^{n} \bar{\kappa}_{i}=|H / g|^{1 / 2}=|\Omega / g|
$$

since $H=\left|H_{i j}\right|=\Omega^{2} / g,[1, \mathrm{p} .260]$. The principal directions for the tensor $H_{i j}$ and those determined by the tensor $\Omega_{i j}$ are identical, since the principal curvature of a principal vector-field can easily be shown equal to the normal curvature of the corresponding line of curvature. Hence we have:

THEOREM 2.1. The spherical curvature of a $V_{n}$ at $P$ is equal to the product of the extreme principal curvatures of vector-fields in $V_{n}$ at $P$, which is the same as the product of principal curvatures of $V_{n}$ at $P$.

Since $S_{n+1}$ is Euclidean, the equations of Gauss are

$$
R_{i j k l}=\Omega_{i k} \Omega_{j l}-\Omega_{i l} \Omega_{j k} .
$$

Multiplying (2.4) by $g^{i k}$ and summing with respect to $i$ and $k$, we obtain

$$
H_{j l}=M \Omega_{j l}+R_{j l},
$$

where $M$ is the mean curvature of $V_{n}$, and where $R_{j l}$ is the Ricci tensor. When $V_{n}$ is a minimal hypersurface, we have $M=0$, and the Ricci tensor is identical 
with the fundamental tensor of $G_{n}$. If $M \neq 0$, we have

$$
H_{i j} p^{i} p^{j}=R_{i j} p^{i} p^{j}
$$

if and only if $v$ is an asymptotic vector-field. If $v$ is a unit asymptotic vectorfield, we notice, from (2.2), (2.6), and the equality

$$
R_{i j} \lambda_{\left.h\right|^{i}} \lambda_{\left.h\right|^{j}}=-\sum_{k=1}^{n} \gamma_{h k},
$$

that the square of the principal curvature of $v$ at $P$ is numerically equal to the sum of the Riemannian curvatures determined by $v$ and $n-1$ other mutually orthogonal unit vectors orthogonal to $v$ at $P$. Hence we have established the following result:

THEOREM 2.2. The square of the principal curvature of an asymptotic vector-field at $P$ in $V_{n}$ is numerically equal to the mean curvature of $V_{n}$ at $P$ for the corresponding asymptotic direction.

The extreme of the principal curvatures $\kappa$ of asymptotic vector-fields at $P$ in $V_{n}$ is defined by

$$
\left|R_{i j}-\kappa^{2} g_{i j}\right|=0
$$

There are $n$ such extreme values corresponding to the principal directions for the Ricci tensor $R_{i j}$. Their product is evidently equal to $|\Omega / g|$, if $V_{n}$ is minimal. Hence we have:

THEOREM 2.3. The principal curvatures of asymptotic vector-fields at $P$ in $V_{n}$ attain their extreme values in the principal directions for the Ricci tensor.

THEOR EM 2.4. The spherical curvature of a minimal $V_{n}$ at $P$ is the product of the principal curvatures of the $n$ vector-fields at $P$ corresponding to the principal directions for the Ricci tensor.

3. The Gaussian curvature. When $n=2, \Gamma$ is called the spherical curvature of a surface $S$ in an ordinary space. It coincides in absolute value with the Gaussian curvature $K$ of $S$. The principal curvature of a vector-field $v$ in $V_{n}$ for $n=2$ coincides in absolute value with the principal curvature of $v$ in $S$, [3]. The extreme principal curvatures of vector-fields in $V_{n}$ for $n=2$ coincide in absolute value with the principal curvatures of $S$. The mean curvature of $V_{n}$ for 
$n=2$ is identical with the Gaussian curvature of $S$. Hence Theorems 2.1 and 2.2 lead directly to the following new interpretations of the Gaussian curvature:

THEOREM 3.1. The Gaussian curvature of $S$ at $P$ is the product of the extreme principal curvatures of vector fields of $S$ at $P$, and is the negative of the square of the magnitude of the Gaussian representation of a unit arc along an asymptotic line from $P$ in $S$.

Let $p^{\alpha}$ and $q^{\alpha}$ be two distinct conjugate vector fields in $S$. Then we have

$$
q^{\beta}=e^{\beta \mu} d_{\alpha \mu} p^{\alpha}
$$$$
(\alpha, \beta, \mu=1,2),
$$

where $d_{a \mu}$ is the second fundamental tensor of $S$. The principal curvatures of the vector-fields $p^{\alpha}$ and $q^{\alpha}$ are respectively equal to

$$
\begin{aligned}
& e \rho_{p}=\left(h_{\alpha \beta} p^{\alpha} p^{\beta} / g_{\alpha \beta} p^{\alpha} p^{\beta}\right)^{1 / 2}, \\
& e \rho_{q}=\left(h g_{\alpha \beta} p^{\alpha} p^{\beta} / g h_{\alpha \beta} p^{\alpha} p^{\beta}\right)^{1 / 2},
\end{aligned}
$$

where $h_{\alpha \beta}$ is the third fundamental tensor of $S$. Hence their product is

$$
\left(e \rho_{p}\right)\left(e \rho_{q}\right)=(h / g)^{1 / 2} .
$$

The expression on the right side of (3.1) is equal to $e K$, where $e$ is +1 or -1 according as $K$ is positive or negative at the point under consideration. At an elliptic point, the principal curvatures of all vector-fields are of the same sign. At a hyperbolic point, the principal curvatures of two vector-fields are different in sign if they lie in different sections separated by the asymptotic lines of $S$. Consequently, the principal curvatures of two conjugate vector-fields have opposite signs, since conjugate directions are separated by the asymptotic directions of the surface. Hence at an elliptic point of $S$, the product of the principal curvatures of two conjugate vector-fields is positive; while at a hyperbolic point of $S$, it is negative. At a parabolic point the normal curvature of any vector-field with respect to any curve is zero. We may consider that every direction in $S$ at a parabolic point is both an asymptotic direction and a principal direction of a vector-field which is to be considered. Hence at a parabolic point the principal curvature of any vector-field is zero; consequently, the product of the principal curvatures of two conjugate vector-fields is zero. Thus the following theorem is proved: 
Theо Ем 3.2. The Gaussian curvature of $S$ at $P$ is the product of the principal curvatures of any two distinct conjugate vector-fields in $S$ at $P$.

The sum of the squares of the principal curvatures of the two conjugate vector-fields is found to be

$$
\left(e \rho_{p}\right)^{2}+\left(e \rho_{q}\right)^{2}=M\left(\kappa_{p}+\kappa_{q}\right)-2 K
$$

where $\kappa_{p}$ and $\kappa_{q}$ are the normal curvatures of the curves of the two fields, and where $M$ is the mean curvature of $S$. By Theorem 3.2 the above equation can be written as

$$
\left(e \rho_{p}+e \rho_{q}\right)^{2}=M\left(\kappa_{p}+\kappa_{q}\right) .
$$

Since the product of the normal radii at a point in conjugate directions is a maximum for characteristic lines, and a minimum for lines of curvature, and since the sum of normal radii in conjugate directions is constant, we obtain from $(3.2)$ the following result:

THEOREM 3.3. The sum of the principal curvatures of two conjugate vectorfields at $P$ is the mean proportional between the mean curvature at $P$ of $S$ and the sum of the normal curvatures in the two conjugate directions at $P$. The square of the sum of the principal curvatures of two conjugate vector-fields at $P$ is a maximum for the principal vector-fields of $S$, and a minimum for the characteristic vector-fields of $S$.

Let $m(m>2)$ directions be such that the angle of two adjoining directions is $2 \pi / m$. Let the principal curvatures of the vector-fields in such directions be denoted by $e \rho_{1}, e \rho_{2}, \cdots, e \rho_{m}$. Then

$$
\frac{1}{m} \sum_{i=1}^{m>2}\left(e \rho_{i}\right)^{2}=\frac{1}{2} M^{2}-K,
$$

since

$$
\frac{1}{m}\left(\sum_{i=1}^{m>2} \kappa_{p_{i}}\right)=\frac{1}{2} M,
$$

where $\kappa_{p_{i}}$ are the normal curvatures of the curves of the corresponding vectorfields. 
THEOREM 3.4. One mth of the sum of the squares of the principal curvatures of $m(>2)$ vector-fields at $P$, such that the angle of two adjoining vectors of these fields at $P$ is $2 \pi / m$, is constant and is the same for any $m$ greater than two. The constant is half of the square of the mean curvature of $S$ minus the Gaussian curvature of $S$ at $P$.

It is easy to prove that the principal direction of a vector-field in $S$ is orthogonal to the curve of the field if and only if the vector-field is an asymptotic field. Let $p^{\alpha}$ be an asymptotic vector-field in $S$. Then its orthogonal trajectories are defined by

$$
d u^{\beta}=e^{\beta \mu} g_{\alpha \mu} p^{\alpha} \text {. }
$$

The principal curvature of the asymptotic vector-field $p^{\alpha}$ is given by

$$
\left(e \rho_{p}\right)=d_{\alpha \beta} p^{\alpha} e^{\beta \mu} g_{\gamma \mu} p^{\gamma} /\left[\left(g_{\alpha \beta} p^{\alpha} p^{\beta}\right)\left(g_{\alpha \beta} e^{\alpha \mu} g_{\gamma \mu} p^{\gamma} e^{\beta \lambda} g_{\sigma \lambda} p^{\sigma}\right)\right]^{1 / 2},
$$

which after simplification becomes

$$
\left(e \rho_{p}\right)=\epsilon^{\beta \mu} d_{\alpha \beta} g_{\gamma \mu} p^{\alpha} p^{\gamma} / g_{\alpha \beta} p^{\alpha} p^{\beta}=\tau_{g},
$$

where $\tau_{g}$ is the geodesic torsion of the curve of the asymtotic vector-field.

THEOREM 3.5. The principal curvature of an asymptotic vector-field at $P$ in $S$ is equal to the geodesic torsion at $P$ of the curve of the field, or simply the torsion at $P$ of the corresponding asymptotic line.

From Theorem 3.1 and Theorem 3.5 we immediately obtain the first part of the theorem of Enneper, that the square of the torsion of a real asymptotic line at a point is equal to the absolute value of the total curvature of the surface at the point. By the second part of the same theorem we notice that the principal curvatures of the asymptotic vector-fields in $S$ are different in sign.

\section{REFERENCES}

1. T. Levi-Civita, The absolute differential calculus, Blackie \& Son, London, 1947. 1949.

2. L. P. Eisenhart, Riemannian geometry, Princeton University Press, Princeton,

3. T. K. Pan, Normal curvature of a vector field, Amer. J. Math. 74 (1952), 955-966. 


\section{PACIFIC JOURNAL OF MATHEMATICS}

\section{EDITORS}

\section{R. U. FOEINSON}

University of California

Berkeley 4, California

E. HewitT

University of Washington

Seattle 5 , Washington
P. P. DILWOR TH

California Institute of Technology

Pasadena 4, California

E. F. BECKENBACH

University of California

Los Angeles 24, California

\section{ASSOCIATE EDITORS}

$\begin{array}{llll}\text { H. BUSEMANN } & \text { P. R. HALMOS } & \text { BØRGE JESSEN } & \text { J. J. STOKER } \\ \text { HERBERT FFDERER } & \text { IIEINZ HOPF } & \text { PAUL LÉVY } & \text { E. G. STRAUS } \\ \text { MARSHALL, IIALI } & \text { R. D. JAMES } & \text { GEORGE PÓLYA } & \text { KÖSAKU YOSIDA }\end{array}$

\section{SPONSORS}

UNIVERSITY OF BRITISH COLUMBIA

CALIFORNIA INSTITUTE OF TECHNOLOGY.

UNIVERSITY OF CAIJIFORNIA, BERKELEY

UNIVERSITY OF CAIJIFORNIA, DAVIS

UNIVERSITY OF CALIFORNIA, LOS ANGELES

UNIVERSITY OF CALIFORNI A, SANTA BARBARA

UNIVERSITY OF NEVADA

OREGON STATE COLLEGE

UNIVERSITY OF OREGON

\author{
UNIVERSITY OF SOU TIERN CALIFORNIA \\ STANFORD RESEARCH INSTITUTE \\ STANFORD UNIVERSITY \\ WASHINGTON STATE COLLEGE \\ UNIVERSITY OF WASHINGTON \\ AMERICAN MATHEMATICAL SOCIETY \\ NATIONAL BUREAU OF STANDARDS, \\ INSTITUTE FOR NUMERICAL ANALYSIS
}

Mathematical papers intended for publication in the Pacific Journal of Mathematics should be typewritten (double spaced), and the author should keep a complete copy. Manuscripts may be sent to any of the editors except Robinson, whose term expires with the completion of the present volume; they might also be sent to M.M. Schiffer, Stanford University, Stanford, California, who is succeeding Robinson. All other communications to the editors should be addressed to the managing editor, E. F. Beckenbach, at the address given above.

Authors are entitled to receive 100 free reprints of their published papers and may obtain additional copies at cost.

The Pacific Journal of Mathematics is published quarterly, in March, June, September, and December. The price per volume (4 numbers) is $\$ 8.00$; single issues, $\$ 2.50$. Special price to individual faculty members of supporting institutions and to individual members of the American Mathematical Society: $\$ 4.00$ per volume; single issues, $\$ 1.25$.

Subscriptions, orders for back numbers, and changes of address should be sent to the publishers, University of California Press, Berkeley 4, California.

Printed at Ann Arbor, Michigan. Entered as second class matter at the Post Office, Berkeley, California.

\section{UNIVERSITY OF CALIFORNIA PRESS • BERKELEY AND LOS ANGELES}




\section{Pacific Journal of Mathematics}

\section{Vol. 3, No. 2 \\ April, 1953}

William George Bade, An operational calculus for operators with spectrum

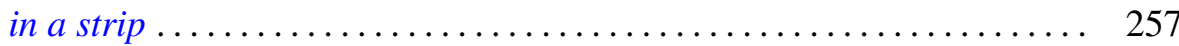

E. F. Beckenbach and Lloyd Kenneth Jackson, Subfunctions of several variables ..................................... 291

David Blackwell, Extension of a renewal theorem ................. 315

L. Carlitz, Some theorems on the Schur derivative ................ 321

Paul Arnold Clement, Generalized convexity and surfaces of negative curvature..................................... 333

Merrill M. Flood, On the Hitchcock distribution problem ............... 369

Watson Bryan Fulks, On the unique determination of solutions of the heat

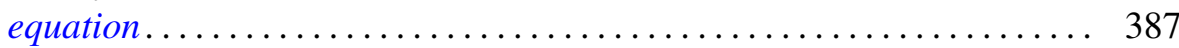

John W. Green, Length and area of a convex curve under affine transformation .................................... 393

William Gustin, An isoperimetric minimax .................. 403

Arthur Eugene Livingston, Some Hausdorff means which exhibit the Gibbs'

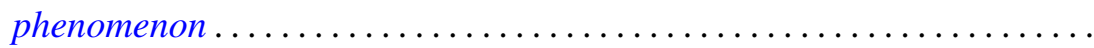

Charles Loewner, On generation of solutions of the biharmonic equation in the plane by conformal mappings ..................... 417

Gábor Szegő, Remark on the preceding paper of Charles Loewner ....... 437

Imanuel Marx and G. Piranian, Lipschitz functions of continuous

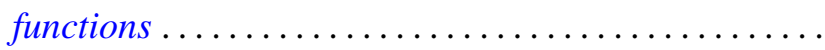

Ting-Kwan Pan, The spherical curvature of a hypersurface in Euclidean space ..

Ruth Lind Potter, On self-adjoint differential equations of second order ...

E. H. Rothe, A note on the Banach spaces of Calkin and Morrey...

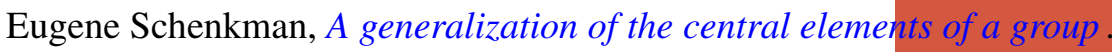

A. Seidenberg, A note on the dimension theory of rings .. . . 